\title{
Incidental pulmonary embolism in cancer patients: clinical characteristics and outcome - a comprehensive cancer center experience
}

This article was published in the following Dove Press journal:

Vascular Health and Risk Management

I4 March 20II

Number of times this article has been viewed

\author{
Hikmat N Abdel-Razeq' \\ Asem H Mansour ${ }^{2}$ \\ Yousef $M$ Ismael $^{\prime}$ \\ 'Department of Internal Medicine, \\ ${ }^{2}$ Department of Radiology, \\ King Hussein Cancer Center, \\ Amman, Jordan
}

Background and objectives: Cancer patients undergo routine imaging studies much more than others. The widespread use of the recently introduced multi-detector CT scanners has resulted in an increasing number of incidentally diagnosed pulmonary embolism (PE) in asymptomatic cancer patients. The significance and clinical outcome of such incidental PE is described.

Methods: Both radiology department and hospital databases were searched for all cancer patients with a diagnosis of incidental PE. CT scans were performed using a 64-slice scanner with a $5.0 \mathrm{~mm}$ slice thickness.

Results: During the study period, 34 patients with incidental PE were identified. The mean age $( \pm \mathrm{SD})$ was $57.7( \pm 12.4)$ years. All patients had active cancer, gastric, lung, colorectal, and lymphomas being the most frequent. Most patients had advanced-stage disease at the time of PE diagnosis; 26 (77\%) patients had stage IV, whereas only 3 patients had stages I or II disease. Twenty-seven (79\%) patients had their PE while undergoing active treatment with chemotherapy $(68 \%)$ or radiotherapy (12\%); none, however, were on hormonal therapy. Most (74\%) patients had their PE diagnosed without history of recent hospital admission. Except for 5 (15\%), all other patients were anticoagulated. With follow-up, 2 patients developed recurrent PE, 2 others had clinical and echocardiographic evidence of pulmonary hypertension, and $9(26 \%)$ died suddenly within 30 days of the diagnosis of incidental PE; 2 of these where among the 5 patients who were not anticoagulated.

Conclusion: Incidental PE in cancer patients is increasingly encountered. Similar to symptomatic PE, many were diagnosed in patients with advanced stage disease and while undergoing active anti-cancer therapy. A significant percentage of patients had recurrent emboli, pulmonary hypertension, and sudden death.

Keywords: pulmonary embolism, incidental, cancer, chemotherapy

\section{Introduction}

Deep vein thrombosis (DVT) and pulmonary embolism (PE), collectively known as venous thromboembolism (VTE), are relatively common. Given its silent nature, the incidence, prevalence, and mortality rates of VTE are probably underestimated. ${ }^{1}$

Cancer and its treatment are recognized risk factors for VTE. Some studies have reported a 6-fold increased risk of VTE in patients with cancer compared with those without. ${ }^{2}$ Active cancer accounts for almost $20 \%$ of all new VTE events occurring in the community. ${ }^{3}$ The risk of VTE varies by cancer type and is especially high among patients with malignant brain tumors, and adenocarcinoma of the ovary, pancreas, colon, stomach, lung, and prostate. ${ }^{4}$
Correspondence: Hikmat Abdel-Razeq Division of Hematology and Medical Oncology, Department of Interna Medicine, King Hussein Cancer Center Queen Rania Al Abdullah Street, PO Box 1269, Amman II94I, Jordan Tel $+962-6-5300460$ ext 1000 Fax +962-6-535300I

Email habdelrazeq@khcc.jo 
Cancer patients undergo routine imaging studies much more than others; these imaging studies, which usually include CT scans of the chest, are done to assess the extent of the disease (staging), assess the response to cancer therapy, or to screen for metastasis. The widespread use of the recently introduced multi-detector CT scanners has resulted in improved resolution and much better visualization even of peripheral pulmonary vessels. Venous thromboembolisms, including PE, in asymptomatic patients are well-recognized clinical entities, and it is believed that most fatal PE are not suspected clinically and are not treated. ${ }^{5,6}$

Many recent reports have addressed the issue of incidentally diagnosed PE; however, few were in cancer patients. The significance and clinical outcome of asymptomatic PE diagnosed incidentally in such high-risk patients are not fully characterized. Treatment decisions are not clear, either. In this report, we will describe the characteristics of 34 incidentally diagnosed $\mathrm{PE}$ in patients undergoing staging and related work-up for cancer.

\section{Materials and methods}

A hospital database (King Hussein Cancer Center, Amman, Jordan) was searched for all patients with a diagnosis of incidental PE, which was defined as radiologically detected PE on imaging studies done for reasons other than confirming or excluding such diagnosis. The radiology database was also queried for all CT reports with similar diagnosis. To further identify our study patients, medical records and radiology reports of all patients were reviewed by at least 2 authors to determine the indication for the CT, tumor type, stage, and anti-cancer therapy particularly in relation to PE development, including surgery, chemotherapy, and radiation therapy. Clinical notes were also reviewed for possible risk factors or clues that would have suggested a diagnosis of PE. Active treatment, when offered, for incidental PE was recorded and final outcomes were identified.

CT was performed using a Philips Briliance-64 slice scanner with a $5.0 \mathrm{~mm}$ slice thickness. The contrast volume was $10 \mathrm{~mL}$ infused at a rate of $3.0 \mathrm{~mL}$ per second.

Descriptive statistical analysis was performed to present patients' demographic and clinical characteristics, treatment rate, and clinical outcome.

\section{Results}

From January 2005 through June 2010, 34 patients, 14 (41\%) males and 20 (59\%) females, with incidental PE were identified. The mean age $( \pm S D)$ of the whole group was $57.7( \pm 12.4)$ years. All patients had a pathologically confirmed diagnosis of cancer; the most frequent cancers were gastric, diagnosed in $6(18 \%)$ patients, followed by lung cancer in $5(15 \%)$, and colorectal and lymphoma in $4(12 \%)$ patients each. The indications for CT scan were: initial staging in $8(24 \%)$, response evaluation in 22 (64\%), and surveillance for metastasis in $4(12 \%)$. The majority of patients had advanced-stage disease at time of PE diagnosis; 26 (77\%) patients had stage IV and 5 (15\%) had stage III disease, whereas only 3 patients had stages I or II disease. Twenty-seven (79\%) patients had their PE while undergoing active treatment with chemotherapy $(68 \%)$ or radiotherapy $(12 \%)$; none, however, were on hormonal therapy (Table 1).

Only 2 patients had a prior diagnosis of DVT and both where on oral anticoagulants. Twenty-five (74\%) patients had their incidental PE diagnosed while ambulatory and without history of recent hospital admission. Following the incidental diagnosis of $\mathrm{PE}$, retrospective evaluation of the patients' medical records showed clinical evidence of DVT in $6(18 \%)$ patients while $18(53 \%)$ had some respiratory symptoms (shortness of breath, chest pain, or hemoptysis alone or in

Table I Patients' characteristics

\begin{tabular}{ll}
\hline Gender & \\
Male & $14(41.2 \%)$ \\
Female & $20(58.8 \%)$ \\
Total & $34(100 \%)$ \\
Age (years) & \\
Range & $27-82$ \\
Mean $( \pm$ SD) & $57.7( \pm 12.4)$ \\
Median & 59 \\
Primary cancer & \\
Gastric & $6(17.6 \%)$ \\
Lung & $5(14.7 \%)$ \\
Colorectal & $4(11.8 \%)$ \\
Lymphoma & $4(11.8 \%)$ \\
Breast & $3(8.8 \%)$ \\
Ovarian & $3(8.8 \%)$ \\
Melanoma & $2(5.9 \%)$ \\
Others & $7(20.6 \%)$ \\
Indication for CT & \\
Initial staging & $4(11.8 \%)$ \\
Response evaluation & $22(64.7 \%)$ \\
Surveillance of metastasis & $8(23.5 \%)$ \\
Stage & \\
I & $1(2.9 \%)$ \\
II & $2(5.9 \%)$ \\
III & $5(14.7 \%)$ \\
IV & $26(76.5 \%)$ \\
Cheologic treatment & \\
Radiotherapy & $23(67.6 \%)$ \\
Hormonal therapy & $4(I 1.8 \%)$ \\
None & $0(0.0 \%)$ \\
\hline & $7(20.6 \%)$ \\
\hline
\end{tabular}


Table 2 Clinical characteristics

\begin{tabular}{ll}
\hline Respiratory symptoms & \\
Shortness of breath & $16(40.0 \%)$ \\
Chest pain & $6(15.0 \%)$ \\
Hemoptysis & $2(5.0 \%)$ \\
None & $16(40.0 \%)$ \\
Immobilization & $10(29.4 \%)$ \\
Clinical evidence of DVT & $6(17.6 \%)$ \\
Hypoxemia & $9(26.5 \%)$ \\
EKG changes & $10(29.4 \%)$ \\
Radiologic features & \\
Central PE & $18(52.9 \%)$ \\
Peripheral PE & $16(47.1 \%)$ \\
\hline
\end{tabular}

Note: ${ }^{\mathrm{B}}$ Because some patients had more than one respiratory symptom, total number is more than 34 .

Abbreviations: DVT, deep vein thrombosis; EKG, electrocardiogram; PE, pulmonary embolism.

combination) at time of incidental PE diagnosis. Tachycardia, electrocardiogram (EKG) changes, and hypoxemia were detected in $16(47 \%), 10(29 \%)$, and $9(26 \%)$, respectively. Pulmonary embolism was central in $18(53 \%)$ while it was detected in a peripheral pulmonary artery in the remaining $16(47 \%)$ patients (Table 2 ).

Following the diagnosis of PE, 29 (85\%) patients were anticoagulated with a low-molecular-weight heparin with or without oral anticoagulant. The 5 patients who were not anticoagulated were also reviewed; all had stage IV disease and 4 were undergoing active cancer treatment with chemotherapy or radiotherapy. Except for 5 (18\%), all patients were treated on an outpatient setting without a need for hospitalization.

The clinical courses of the patients were followed; 2 patients developed recurrent PE documented on imaging studies following clinical presentation suggestive of PE, while 2 others had clinical and echocardiographic evidence of pulmonary hypertension. Surprisingly, 9 (26\%) died suddenly within 30 days of the diagnosis, 2 of whom where among the 5 patients who were not anticoagulated (Table 3).

\section{Discussion}

Incidental PE is not an uncommon diagnosis in clinical practice, especially in high-risk patients like those with cancer. Depending on the patient population studied, the diagnostic criteria, and technique used, the incidence of incidental PE varies, some studies describing an incidence as high as $4.3 \%{ }^{7,8}$

In a recent study, the medical records of 1921 consecutive cancer patients starting chemotherapy were reviewed; overall, there were $101(5.3 \%)$ VTE, 62 (3.2\%) of which were
Table 3 Treatment and outcome

\begin{tabular}{ll}
\hline Treatment for PE & \\
Anticoagulation & $29(85.3 \%)$ \\
No anticoagulation & $5(14.7 \%)$ \\
Outcome & \\
$\quad$ Recurrent PE & $2(5.9 \%)$ \\
Pulmonary hypertension & $2(5.9 \%)$ \\
$\quad$ Sudden death & $9(26.5 \%)$ \\
Treatment given & \\
Inpatient & $15(44.1 \%)$ \\
$\quad$ Outpatient & $19(55.9 \%)$ \\
\hline Abbreviation: PE, pulmonary embolism. &
\end{tabular}

incidental; more than one-third (24/62) were PE. ${ }^{9}$ In another retrospective study that included 435 consecutive staging CT chest, abdomen, and pelvis scans performed on a variety of cancer patients, PE was detected in $13(3.3 \%)$ while $6.8 \%$ had unsuspected iliofemoral and 1.2\% had unsuspected common iliac DVT; both DVT and PE were more common in hospitalized patients $(P=0.002,0.004$; relative risk $[\mathrm{RR}]=1.6,2.1$, respectively) and in those with advanced disease $(P=0.001,0.01 ; \mathrm{RR}=2.2,1.8$, respectively $) .{ }^{10}$ The variation in incidence rate of unsuspected PE can be attributed to the heterogeneity of the patient population studied. However, the technique of the CT scan, including slice thickness, plays a significant role, too. A recently published meta-analysis of 12 studies addressing incidental, asymptomatic PE that included more than 10,000 patients found a prevalence of $2.0 \%$ (95\% CI 1.0, 3.4) when using CT scans with $\geq 5 \mathrm{~mm}$ slice thickness compared with a prevalence of $3.0 \%$ (95\% CI 2.0, 4.0) when using scans with $<5 \mathrm{~mm}$ slice thickness. ${ }^{11}$ This issue was also addressed in another study, in which incidental PE was more frequent with a $1 \mathrm{~mm}$ slice thickness scans $(6 \%)$ comparison with the 2 to $3 \mathrm{~mm}$ slice thickness scans (4.7\%), although this difference was not statistically significant. ${ }^{12}$

Silent PE following a diagnosis of DVT is not the same as incidental PE under discussion in this report. Patients with DVT, especially high-risk ones, might develop a silent PE that can be detected by imaging studies in a relatively high percentage of such patients. In a systemic review of published literature, silent PE was diagnosed in 1665 (32\%) of 5233 patients with DVT. The incidence of silent PE was higher with proximal DVT than with distal DVT. ${ }^{13}$

Previous work in cancer patients with symptomatic VTE highlighted certain clinical variables that can identify cancer patients at higher risk of developing VTE. First, the risk of VTE is highest among certain cancer types like brain, ovarian, gastric, and pancreatic tumors, ${ }^{14,15}$ but lower in sites such as skin, breast, and thyroid. ${ }^{16,17}$ Second, the risk of 
VTE is highest during the first 3 to 6 months after the initial diagnosis of cancer. ${ }^{18}$ Third, the risk varies with the stage of the disease, being much higher with advanced stage than with early stage disease. ${ }^{18}$ Fourth, the risk is also higher among cancer patients on active treatment with chemotherapy or radiotherapy. ${ }^{19}$

Similar to what has been reported in symptomatic VTE in cancer patients discussed above, most of our incidental PE were diagnosed in patients undergoing active cancer treatment with chemotherapy and radiation therapy and during the early phases of their cancer, as shown by the fact that almost $25 \%$ had such diagnosis while undergoing initial staging work-up and 64\% had their incidental PE diagnosed while undergoing imaging studies to evaluate response to active treatment. Additionally, we may conclude that other important tumor characteristics such as disease stage are also important factors in incidental PE. The presence of advanced stage disease, identified in more than $75 \%$ of our patients, is associated with higher risk of PE. However, given the relatively small number of reported cases of incidental PE and the selection bias related to higher frequency of imaging studies done for certain tumor types, by virtue of the disease itself or the treatment given for lymphomas and lung cancer for example, it would be difficult to state that incidental PE is more common in certain tumor types or to suggest or recommend routine evaluation or even closer follow-up.

The management of incidental PE might not be clear in the mind of practicing physicians. Current clinical guidelines published by the American College of Chest Physicians recommend the same initial and same long-term anticoagulant therapy for patients who are unexpectedly found to have asymptomatic PE as for patients with symptomatic PE. ${ }^{20}$ However, others argued that a similar outcome can be achieved without anticoagulation. ${ }^{21}$ In one study, Engelke et al retrospectively assessed the outcome of PE treatment in a group of 96 patients, many of whom had unexpected PE and many were cancer patients. Forty-nine (51\%) patients received therapeutic anticoagulation while $26(27 \%)$ received no treatment; 21 (22\%) patients, however, were given only a prophylactic dose of anticoagulation. PE severity was higher in patients with therapeutic anticoagulation than in those without $(P<0.001)$. Bleeding complications were more frequent with therapeutic anticoagulation (2 early deaths, 5 major nonfatal hemorrhages) than without (1 minor hemorrhage; $P=0.037$ ). There were 8 early deaths, 7 in the therapeutic anticoagulation group and 1 in the group without anticoagulation $(P=0.037)$. The authors concluded that clinically unsuspected PE may remain undetected at routine chest $\mathrm{CT}$ and such patients have favorable short-term outcome without therapeutic anticoagulation. ${ }^{21}$ However, physicians should be careful when applying such findings in clinical practice.

Though defined as incidental, our study showed that with close questioning or review of their medical records, some of our study patients had symptoms or signs that could have been attributed to PE. Among our cases, respiratory symptoms in form of shortness of breath, chest pain, and hemoptysis were identified in $40 \%$, and $25 \%$ or more of the patients had hypoxemia or EKG changes.

Our study also adds to the growing evidence that ambulatory cancer patients can be at risk for VTE. In our study, $76 \%$ of the patients had their PE without history of recent hospitalization. Non-hospitalized, ambulatory cancer patients on active anti-cancer therapy can be at high risk for VTE, too; current guidelines, however, do not recommend anticoagulant prophylaxis for such patients. Khorana et al tried to establish a risk assessment model for VTE prophylaxis in ambulatory cancer patients after the initiation of chemotherapy. The site of the primary tumor, platelet count, leukocyte counts, hemoglobin level, use of erythropoiesis stimulating agents, and body mass index were found to be predictive factors for the occurrence of VTE. ${ }^{22}$ In another recent study by the Vienna Cancer and Thrombosis Study Group, investigators reported that elevated serum P-selectin levels predicted VTE in 687 newly diagnosed ambulatory cancer patients. The cumulative probability of VTE after 6 months of follow-up was $11.9 \%$ in patients with high serum $\mathrm{P}$-selectin compared with $3.7 \%$ in those with low levels $(P=0.002) .^{23}$

Our patient population in this study, and others similar previously, are too small to draw firm conclusions about these associated risk factors. More work is needed to further address the issue of VTE in ambulatory cancer patients.

The retrospective nature of our study and the active anticoagulation of the majority ( $85 \%$ ) of our study patients did not allow us to trace the clinical relevance of incidental PE. Previous studies have shown that patients with cancer have more than a 4-fold higher risk of dying after a acute thrombotic event than cancer patients without. ${ }^{24,25}$ In one study, Sørensen et al found that 1-year survival rate for cancer patients with thrombosis was $12 \%$ compared with $36 \%$ in cancer patients without VTE $(P<0.001) .{ }^{26}$ Though our study cannot confirm a similar negative impact of incidental PE, our findings offer some clues to suggest a similar poor outcome; $25 \%$ of our patients had sudden death while 
another $12 \%$ had recurrent PE or pulmonary hypertension. Few other studies have attempted to provide clues on the natural history of incidental PE; however, many were in non-cancer patients. In one study, and at clinical follow-up that varied from 2 days to 24 months, symptomatic VTE events occurred in 4 of 12 patients in whom incidental PE were not identified at the time of CT scan. ${ }^{27}$ In another study, Storto et al found that progression of PE occurred at follow-up CT scan in 1 of 4 patients in whom PE was not identified at the time of the original imaging study. None of these patients were anticoagulated for their incidental PE. ${ }^{28}$ In a more recent study, O'Connell et al found that cancer patients with incidental PE had an increased risk of death (hazard ratio $1.79 ; 95 \%$ CI 1.10, 2.90) and that such incidental $\mathrm{PE}$ appeared to confer a poorer survival (5 vs 14 months; $P=0.0009){ }^{29}$

Our study is not without limitations. Alhough it is among the largest studies to include cancer patients with incidental PE, the small number of patients studied and its retrospective nature may not allow reliable conclusions. Currently, our group is prospectively collecting data on unsuspected PE diagnosed in CT studies done for reasons other than diagnosing PE. The prospective nature of this ongoing study will allow us to draw conclusions on clinical and other associated risk factors, including some of those addressed in symptomatic PE.

In conclusion, incidental PE in cancer patients is increasingly encountered. Similar to symptomatic PE, many were diagnosed in patients with advanced stage disease and while undergoing active treatment with chemotherapy. Clinical outcomes, in the form of recurrent emboli, pulmonary hypertension, and sudden death, appear to be similar to symptomatic PE.

\section{Acknowledgment}

The authors would like to thank Ms Haifa Al-Ahmad, Mrs Alice Haddadin, and Dr Luna Zaru for their help in preparing this manuscript.

\section{Disclosure}

The authors declare no conflicts of interest.

\section{References}

1. Kniffin WD Jr, Baron JA, Barrett J, Birkmeyer JD, Anderson FA Jr. The epidemiology of pulmonary embolism and deep venous thrombosis in the elderly. Arch Intern Med. 1994;154:861-866.

2. Heit JA, Silverstein MD, Mohr DN, Petterson TM, O'Fallon WM, Melton LJ 3rd. Risk factors for deep vein thrombosis and pulmonary embolism: a population-based case-control study. Arch Intern Med. 2000;160:809-815.
3. Heit JA, O'Fallon WM, Petterson TM, et al. Relative impact of risk factors for deep vein thrombosis and pulmonary embolism: a population-based study. Arch Intern Med. 2002;162:1245-1248.

4. Thodiyil PA, Kakkar AK. Variation in relative risk of venous thromboembolism in different cancers. Thromb Haemost. 2002;87:1076-1077.

5. Ryu JH, Olson EJ, Pellikka PA. Clinical recognition of pulmonary embolism: problem of unrecognized and asymptomatic cases. Mayo Clin Proc. 1998;73:873-879.

6. Pineda LA, Hathwar VS, Grant BJ. Clinical suspicion of fatal pulmonary embolism. Chest. 2001;120:791-795.

7. Engelke C, Manstein P, Rummeny EJ, Marten K. Suspected and incidental pulmonary embolism on multidetector-row CT: analysis of technical and morphological factors influencing the diagnosis in a cross-sectional cancer centre patient cohort. Clinical Radiology. 2006; 61:71-80.

8. Sebastian AJ, Paddon AJ. Clinically unsuspected pulmonary embolism - an important secondary finding in oncology CT. Clinical Radiology. 2006;61:81-85.

9. Di Nisio M, Ferrante N, De Tursi M, et al. Incidental venous thromboembolism in ambulatory cancer patients receiving chemotherapy. Thromb Haemost. 2010;104:1049-1054.

10. Browne AM, Cronin CG, English C, NiMhuircheartaigh J, Murphy JM, Bruzzi JF. Unsuspected pulmonary emboli in oncology patients undergoing routine computed tomography imaging. J Thorac Oncol. 2010;5:798-803.

11. Dentali F, Ageno W, Becattini C, et al. Prevalence and clinical history of incidental, asymptomatic pulmonary embolism: a meta-analysis. Thromb Res. 2010;125:518-522.

12. Ritchie G, McGurk S, McCreath C, Graham C, Murchison JT. Prospective evaluation of unsuspected pulmonary embolism on contrast enhanced multidetector CT (MDCT) scanning. Thorax. 2007; 62:536-540

13. Stein PD, Matta F, Musani MH, Diaczok B. Silent pulmonary embolism in patients with deep venous thrombosis: a systematic review. $\mathrm{Am} \mathrm{J}$ Med. 2010;123:426-431.

14. Sallah S, Wan JY, Nguyen NP. Venous thrombosis in patients with solid tumors: determination of frequency and characteristics. Thromb Haemost. 2002;87:575-579.

15. Chew HK, Wun T, Harvey D, Zhou H, White RH. Incidence of venous thromboembolism and its effect on survival among patients with common cancers. Arch Intern Med. 2006;166:458-464.

16. Andtbacka RH, Babiera G, Singletary SE, et al. Incidence and prevention of venous thromboembolism in patients undergoing breast cancer surgery and treated according to clinical pathways. Ann Surg. 2006;243:96-101.

17. Chew HK, Wun T, Harvey DJ, Zhou H, White RH. Incidence of venous thromboembolism and the impact on survival in breast cancer patients. J Clin Oncol. 2007;25:70-76.

18. Blom JW, Doggen CJ, Osanto S, Rosendaal FR. Malignancies, prothrombotic mutations, and risk of venous thrombosis. JAMA. 2005;293:715-722.

19. Haddad TC, Greeno EW. Chemotherapy-induced thrombosis. Thromb Res. 2006;118:555-768.

20. Kearon C, Kahn SR, Agnelli G, et al. Antithrombotic therapy for venous thromboembolic disease: American College of Chest Physicians Evidence-Based Clinical Practice Guidelines, 8th ed. Chest. 2008; 133:454-545.

21. Engelke C, Rummeny EJ, Marten K. Pulmonary embolism at multi-detector row CT of chest: one year survival of treated and untreated patients. Radiology. 2006;239:563-575.

22. Khorana AA, Kuderer NM, Culakova E, Lyman GH, Francis CW. Development and validation of a predictive model for chemotherapyassociated thrombosis. Blood. 2006;111:4902-4907.

23. Ay C, Simanek R, Vormittag R, et al. High plasma levels of soluble P-selectin are predictive of venous thromboembolism in cancer patients: results from the Vienna Cancer and Thrombosis Study (CATS). Blood. 2008;112:2703-2708. 
24. Carson JL, Kelley MA, Duff A, et al. The clinical course of pulmonary embolism. N Engl J Med. 1992;326:1240-1245.

25. Prandoni P, Lensing AW, Cogo A, et al. The long-term clinical course of acute deep venous thrombosis. Ann Intern Med. 1996;125:1-7.

26. Sørensen HT, Mellemkjaer L, Olsen JH, Baron JA. Prognosis of cancers associated with venous thromboembolism. $N$ Engl J Med. 2000;343:1846-1850.

27. Gladish GW, Choe DH, Marom EM, Sabloff BS, Broemeling LD, Munden RF. Incidental pulmonary emboli in oncology patients: prevalence, CT evaluation, and natural history. Radiology. 2006;240: 246-255.
28. Storto ML, Di Credico A, Guido F, Larici AR, Bonomo L. Incidental detection of pulmonary emboli on routine MDCT of the chest. AJR Am J Roentgenol. 2005;184:264-267.

29. O'Connell C, Razavi P, Ghalichi M, et al. Unsuspected pulmonary emboli adversely impact survival in patients with cancer undergoing routine staging MDCT scanning. J Thromb Haemost. 2011;9:305-311.

\section{Publish your work in this journal}

Vascular Health and Risk Management is an international, peerreviewed journal of therapeutics and risk management, focusing on concise rapid reporting of clinical studies on the processes involved in the maintenance of vascular health; the monitoring, prevention and treatment of vascular disease and its sequelae; and the involvement of metabolic disorders, particularly diabetes. This journal is indexed on PubMed Central and MedLine. The manuscript management system is completely online and includes a very quick and fair peer-review system, which is all easy to use. Visit http://www.dovepress.com/ testimonials.php to read real quotes from published authors. 